\title{
Mehr Transparenz und Schutz der Integrität
}

\section{Jörg Baumann}

General Counsel und stv. Geschäftsführer Swiss Medtech

\author{
Im Swiss Medtech-Kodex sind seit Juni 2017 die Interaktionen der Mitgliedsunter- \\ nehmen des Verbands mit medizinischen Fachkräften und Einrichtungen geregelt. \\ Im Fokus steht u.a. die Unterstützung von medizinischen Fachkräften für die Teil- \\ nahme an Produktschulungen oder an von Dritten organisierten Bildungsver- \\ anstaltungen. Weiter hat die Branche am 1. Januar 2018 Transparenzrichtlinien zur \\ Dokumentation und Offenlegung von Ausbildungszuwendungen eingeführt.
}

Inwieweit sind Spenden, Geschenke, gesponserte Veranstaltungen, finanzielle Zuschüsse durch die Industrie an Fachpersonen im Gesundheitswesen oder die Abgabe von Gratismustern rechtlich erlaubt und ethisch vertretbar? Wo liegen die Grenzen der Zulässigkeit in der Interaktion zwischen Medtech-Firmen und medizinischen Fachpersonen bzw. Einrichtungen? Die Zusammenarbeit kann verschiedenste Facetten haben. Gemeinsames Ziel ist dabei immer, mehr Menschen Zugang zu sicheren, innovativen und verläss-

\section{Wo liegen die Grenzen der Zulässigkeit in der Interaktion zwischen Medtech-Firmen und medizinischen Fachpersonen bzw. Ein- richtungen?}

lichen Produkten sowie zugehörigen Leistungen zu ermöglichen, beispielsweise durch ständige Verbesserung und Weiterentwicklung medizinischer Technologien und Anwendungen. Die von den Unternehmen geleistete Unterstützung von medizinischen Forschungs- und Bildungsprojekten trägt dazu bei, die klinischen Fähigkeiten medizinischer Fachpersonen zu optimieren. Auch helfen angemessene Instruktionen, Bildung, Schulungen und Serviceleistungen dabei, eine sichere und wirksame Nutzung der Medizinprodukte zu gewährleisten.

Ein Aspekt der Zusammenarbeit ist der Öffentlichkeit gegenüber jedoch zunehmend schwieriger zu vertreten: wenn Unternehmen die Kosten einzelner medizinischer Fachkräfte/Kunden für die Teilnahme an von Dritten organisierten Bildungskonferenzen übernehmen (wie Anmeldegebühren sowie Reise- und Übernachtungskosten).

\begin{abstract}
Die wichtigsten Neuerungen betreffend Zuwendungen und Spenden

Zuwendungen dürfen ausschliesslich an juristische Personen, d.h. medizinische Einrichtungen oder professionelle Konferenzveranstalter gezahlt werden, nicht an Einzelpersonen.

Für Zuwendungen wird ein schriftlicher Vertrag mit der medizinischen Einrichtung erforderlich, in dem Zweck, Nutzung und Pflichten der Begünstigten festgelegt sind.

Unternehmen werden zwar nicht berechtigt sein, Einzelpersonen als Empfänger der Zuwendungen für von Dritten organisierte Bildungskonferenzen zu benennen. Sie dürfen jedoch generell den Empfängertyp festlegen.

Ausbildungszuwendungen werden auf einer elektronischen Plattform gemäss Transparenzregeln offengelegt.

Unternehmen sollen über ein unabhängiges Prüfungsverfahren verfügen, um über Zuwendungs- und Spendenanträge zu entscheiden. Dies gilt auch für Ausbildungszuwendungen an professionelle Kongressveranstalter, die unabhängig von medizinischen Einrichtungen tätig sind.
\end{abstract}

\section{Ausbildungszuwendungen konkret definieren}

Mit dem am 12. Juni 2017 von Swiss Medtech in Kraft gesetzten Branchen-Kodex wird die Verpflichtung geschaffen, medizinische Ausbildung ausschliesslich über konkret definierte Arten von Zuwendungen zu unterstützen (siehe Kasten). Hiermit sollen in dieser Beziehung gerechtfertigte Zuwendungen zwischen Branche und medizinischen Fachkräften geschützt werden, so dass diese nicht als unzulässige geldwerte Vorteile oder illegale finanzielle Verbindungen interpretiert werden können.

Der Kodex von Swiss Medtech lehnt sich an denjenigen des europäischen Dachverbands, «MedTech Europe 
Code of Ethical Business Practice», an und betrifft alle Veranstaltungen, die durch die Mitgliedsunternehmen des Verbands unterstützt oder organisiert werden. Er regelt die Unterstützung von medizinischen Fachkräften für die Teilnahme an Produktschulungen oder an von Dritten organisierten Bildungsveranstaltungen. Neu ist seit dem 1. Januar 2018 hier eine direkte Finanzierung der Teilnahme von medizinischen Fachpersonen an Bildungskonferenzen durch Medtech-Firmen nicht mehr möglich. Vielmehr dürfen Zuwendungen nur noch an medizinische Einrichtungen oder professionelle Konferenzveranstalter ausgerichtet werden. Zusätzlich werden Transparenzrichtlinien zur Dokumentation und Offenlegung von Ausbildungszuwendungen eingeführt.

\section{Neu ist eine direkte Finanzierung der Teil- nahme von medizinischen Fachpersonen an Bildungskonferenzen durch Medtech-Firmen nicht mehr möglich.}

Weiter enthält der Kodex spezifische Regeln für bestimmte Veranstaltungstypen (Konferenzen, Produktund Anwendungsschulungen etc.), für Forschungs- und Beratervereinbarungen, Zuwendungen und Spenden, Muster und Demonstrationsprodukte, Material für die fachliche Weiterbildung und Geschenke von geringfügigem Wert.

\section{Unabhängige Behandlungsentscheide sicherstellen}

Mit der Regelung jeglicher Interaktionen zwischen Mitgliedsunternehmen von Swiss Medtech und medizinischen Fachkräften bzw. Einrichtungen ist gemäss Kodex sicherzustellen, dass Kliniken, Ärzte, Labortechniker, Pflegekräfte und Krankenhäuser/Kliniken unabhängige Entscheide für medizinische Behandlungen fällen können, ohne dass dabei ein falscher Ein- druck durch Unterstützung und Tätigkeiten der Branchenfirmen entsteht. Zudem sollen Interaktionen in einer angemessenen Umgebung stattfinden, und die Vergütung von Leistungen durch medizinische Fachkräfte soll gemäss dem Prinzip der Ausgewogenheit erfolgen.

\section{Der neue Kodex soll gewährleisten, dass} Medizintechnikunternehmen und ihre Vertreter gemäss höchsten ethischen Standards handeln.

Zweck des Swiss Medtech-Kodex zum ethischen Geschäftsverhalten ist, die Integrität und Reputation aller an der Entwicklung innovativer Medizintechnik beteiligten Parteien noch besser zu schützen. Dabei handelt es sich um eine proaktive, stringente Selbstregulierung durch die Branche, die über die geltende Gesetzgebung hinausgeht. Ziel ist die Förderung eines ausgewogenen regulatorischen Umfelds, das die Medtech-Industrie dabei unterstützt, die zunehmenden Anforderungen des Gesundheitswesens und die steigenden ethischen Erwartungen ihrer Interessenspartner zu erfüllen.

\section{Nach höchsten ethischen Standards handeln}

Der neue Kodex soll gewährleisten, dass MedtechUnternehmen und ihre Vertreter gemäss höchsten ethischen Standards handeln. Er dient dazu, die positive Aussenwahrnehmung bzw. das öffentliche Vertrauen und Verständnis für die wertvollen Beiträge aus der Zusammenarbeit mit medizinischen Fachkräften zu stärken.

Der Kodex und weitere Informationen sind auf der Website von Swiss Medtech unter https://swiss-medtech.ch/ de/web/swiss-medtech-website/legal-compliancel verfügbar. 